$>$ Les tumeurs stromales gastro-intestinales (GIST) étaient très souvent méconnues jusqu'en 1998, alors que ce sont les sarcomes les plus fréquents chez l'homme. Elles dérivent des cellules de Cajal et se développent dans le tractus digestif préférentiellement à partir de l'estomac ou de l'intestin grêle. Elles sont caractérisées par une expression très fréquente de KIT (CD117), et des mutations de KIT ou de PDGFRA (alphatype plateled-derived growth factor receptor) sont observées dans $85 \%$ des cas. Plus de 150 mutations différentes ont été décrites. Elles sont responsable d'une activation de ces récepteurs à activité tyrosine kinase en l'absence de leurs ligands spécifiques. La détection de ces mutations peut aider à confirmer le diagnostic ou évaluer le pronostic. Les mutations sont également prédictives de la réponse au traitement par les inhibiteurs de KIT. Ainsi, la présence d'une duplication de l'exon 9 de KIT est une indication à un doublement de la posologie de l'imatinib dans les GIST métastatiques, et les GIST ayant une mutation ponctuelle p.D842V de PDGFRA sont résistantes à ce traitement. Cet article de synthèse décrit les principales caractéristiques des GIST, en insistant sur les apports de l'étude de ces tumeurs et de leurs modèles cellulaires dans le domaine de la cancérologie. <

Les tumeurs stromales gastro-intestinales (GIST) sont des tumeurs mésenchymateuses qui peuvent se développer tout le long du tractus digestif. Elles sont caractérisées par leur aspect histologique, l'expression quasi constante du CD117 (KIT) ${ }^{1}$ et la fréquence importante de mutations activatrices des gènes KIT ou PDGFRA.

Vignette (Photo (c) Inserm-Bernard Vandenbunder)

${ }^{1}$ KIT est le récepteur du facteur de croissance SCF (stem cell factor), aussi appelé ligand KIT, initialement caractérisé par son rôle prédominant dans le système hématopoïétique.

\section{Les tumeurs} stromales gastrointestinales (GIST)

\section{À l'avant-garde des thérapies ciblées antitumorales}

Jean-François Émile

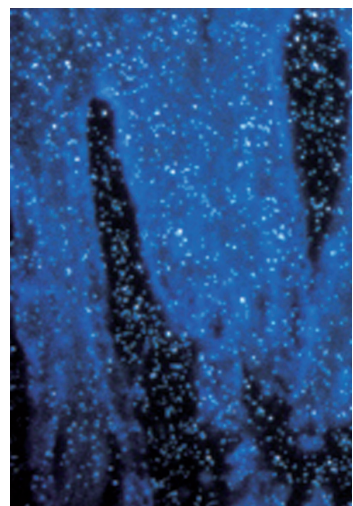

Université de Versailles, EA4340, service de pathologie, hôpital Ambroise Paré, assistance publique-hôpitaux de Paris, 9, avenue Charles de Gaulle, 92104 Boulogne, France. jean-francois.emile@uvsq.fr

Elles ont été décrites il y a une trentaine d'années [1], mais l'identification précise de ces tumeurs n'a été possible en pratique courante qu'à partir de 1998, grâce aux travaux d'une équipe japonaise montrant l'importance de l'oncogène KIT dans la genèse de ces tumeurs [2]. Le succès thérapeutique du traitement ciblé par l'imatinib² [3], publié seulement trois années après la découverte du rôle oncogénique de KIT, a été la première démonstration de l'efficacité d'une thérapie ciblée dans les tumeurs solides. Le nombre de publications sur les GIST depuis ces deux découvertes (Figure 1) atteste de l'intérêt extraordinaire de ce cancer rare pour les oncologues. Nous exposerons dans le texte qui suit les caractéristiques histologiques, moléculaires et cliniques des GIST, en insistant plus particulièrement sur les aspects ayant contribué à l'émergence de nouveaux concepts en oncologie. Nous présenterons également quelques résultats d'études de modèles cellulaires de GIST et les questions qu'ils soulèvent concernant l'activation des récepteurs de type tyrosine kinase et leur inhibition médicamenteuse.

2 L'imatinib, ou Glivec (initialement appelé CGP57148, STI571 puis l'imatinib mésylate), un dérivé de la 2-aminopyrimidine, a été initialement développé pour s'opposer à l'action kinase constitutive de la protéine de fusion $B C R-A B L$, produit de la translocation $(9 ; 22)$ inductrice de la leucémie myéloïde chronique. L'imatinib agit au niveau de la poche à ATP de la portion $A B L$ de manière sélective, inhibe également l'activité tyrosine kinase du PDGF, mais n'a aucun effet inhibiteur sur l'activité tyrosine kinase d'autres récepteurs comme le VEGF-R et $\varepsilon$ GF-R. Cette sélectivité a conduit rapidement à l'introduction de cet inhibiteur en clinique en 1996 (voir [42]). 


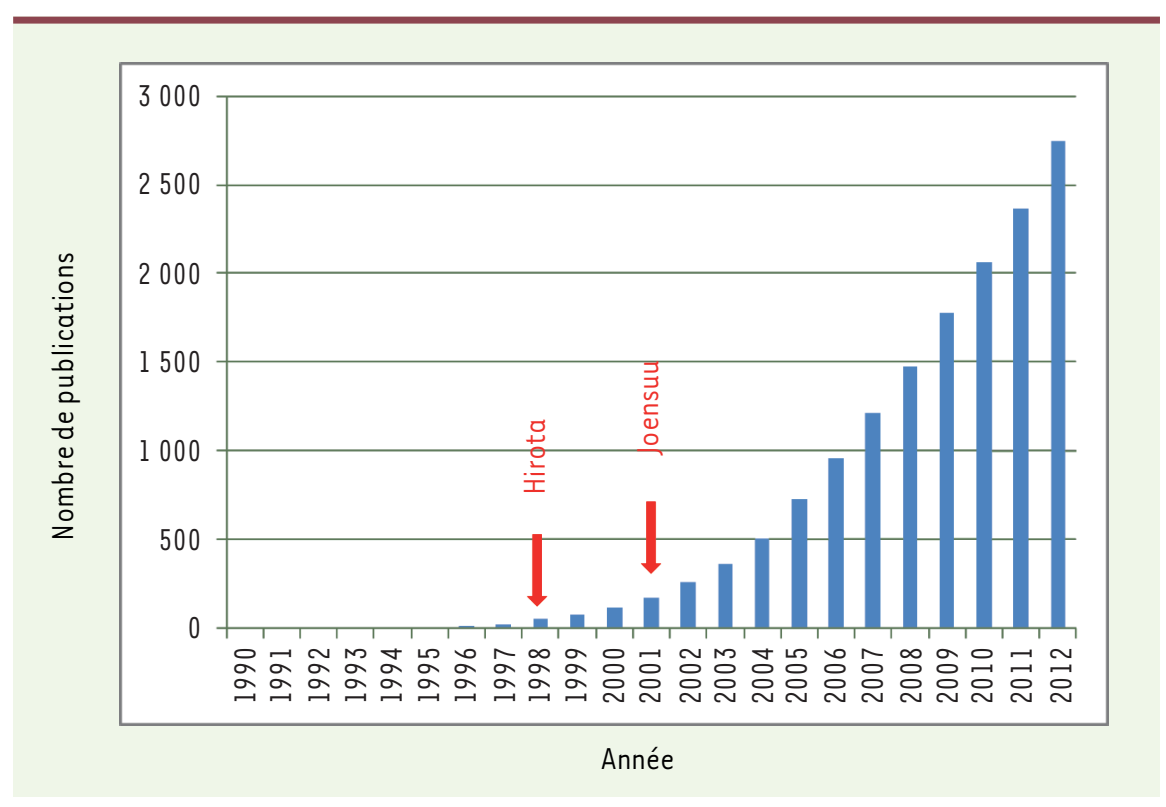

Figure 1. Publications sur les GIST. Le nombre total de publications sur les GIST* exprimé en fonction des années démontre l'intérêt récent de la communauté scientifique pour ces tumeurs. Après la première description en 1983, les publications de Hirota et al. [2] et Joensuu et al. [3] ont été des étapes majeures dans l'initiation des travaux de recherche sur les GIST. *Interrogation pubmed au 5 janvier 2013 : GIST (Title/Abstract) AND (stromal[Title/ Abstract] OR gastrointestin* (Title/ Abstract).

difficile et, en France, une relecture systématique des lames est réalisée dans le cadre du Réseau sarcomes soutenu par l'Institut national du

\section{Les tumeurs stromales gastro-intestinales (GIST)}

Les GIST surviennent plus fréquemment à l'âge adulte, avec un âge médian d'environ 60 ans et une fréquence équivalente dans les deux sexes [4]. Elles se développent souvent à partir de l'estomac (60\%) ou de l'intestin grêle (25\%). Leur taille au moment du diagnostic est très variable (Figure 2), de moins de un cm (micro-GIST) jusqu'à une quarantaine de $\mathrm{cm}$. Les GIST sont des tumeurs rares, avec une incidence annuelle de 15 à 20 pour 100000 habitants, mais elles sont néanmoins les sarcomes les plus fréquents chez l'humain [5].

Ces tumeurs sont généralement peu symptomatiques car elles se développent principalement vers l'extérieur de la paroi digestive. Le diagnostic repose sur l'étude histologique qui met en évidence un aspect le plus souvent fusiforme ou épithélioïde des cellules tumorales (Figure 3) [6]. Le diagnostic nécessite également de réaliser une étude immunohistochimique avec des anticorps spécifiques des protéines KIT et DOG $-1^{3}$ (discovered on GIST-1) (Figure 3). La positivité des cellules tumorales pour chacun de ces deux marqueurs est d'environ $95 \%$ [7]. Les GIST correspondent à une prolifération de cellules interstitielles de Cajal (ou de leur progéniteurs). Il s'agit de cellules fusiformes ou étoilées, peu abondantes, réparties au sein de la musculeuse du tractus digestif. Elles sont douées d'une activité électrique pulsatile spontanée (pacemaker) qui active le péristaltisme du tube digestif. Des GIST spontanées ont été décrites chez plusieurs autres mammifères (chien, chat, furet) [8] et il existe également des modèles de souris génétiquement modifiées qui développent ce type de tumeurs.

Avant les années 2000, les GIST étaient, dans la grande majorité des cas, confondues avec d'autres tumeurs, telles que les léiomyomes, les léiomyosarcomes et les schwannomes $[6,7]$. L'utilisation actuelle de marqueurs très sensibles et spécifiques (KIT et DOG-1) permet maintenant un diagnostic plus fiable. Ce diagnostic reste toutefois parfois

${ }^{3}$ Protéine de fonction inconnue. cancer (INCa) depuis 2010.

La très grande majorité des GIST sont sporadiques mais, comme pour toute tumeur, il existe quelques prédispositions génétiques. La plus fréquente est la neurofibromatose de type $1^{4}$. Chez ces patients, le risque de développer une GIST au cours de la vie est d'environ $7 \%$, et les tumeurs sont souvent multiples et le gène KIT n'est pas muté [9]. II existe d'autres prédispositions génétiques, plus rares, telles que la triade de Carney qui associe GIST, ganglioneurome ${ }^{5}$ [43] et chondrome pulmonaire, et le syndrome de Stratikis-Carney dans lequel des mutations germinales de SDHB (succinate dehydrogenase complex, subunit $B$ ) ont été détectées [10]. Enfin, il existe des formes familiales avec des mutations de KIT ou de PDGFRA [11] (voir plus loin).

Les formes dites pédiatriques de GIST semblent constituer une entité particulière. Elles surviennent chez l'enfant ou l'adulte jeune, préférentiellement de sexe féminin, et sont localisées principalement à l'estomac. Leur évolution est généralement lente, avec possibilité de métastases ganglionnaires [12]. Elles ne présentent habituellement pas de mutation de KIT ni de PDGFRA, et il peut exister une perte d'expression de SDHB en immunohistochimie.

${ }^{4}$ La neurofibromatose 1 (ou maladie de von Recklinghausen) est une maladie autosomique dominante qui atteint environ 1 individu sur 4000 , caractérisée par des neurofibromes multiples, des taches café au lait, des lentigines axillaires ou inguinales, des anomalies squelettiques et une prédisposition aux leucémies et aux tumeurs gliales. Ces tumeurs se développent à partir des nerfs. Le gène a été localisé au chromosome 17 en 1987.

${ }^{5}$ Les paragangliomes ( $\mathrm{PGL}$ ) sont des tumeurs neuroendocrines rares qui se développent aux dépens du tissu paraganglionnaire. Celui-ci est issu de la migration des cellules de la crête neurale et constitue le système nerveux sympathique et parasympathique. (reproduit de [43]). 

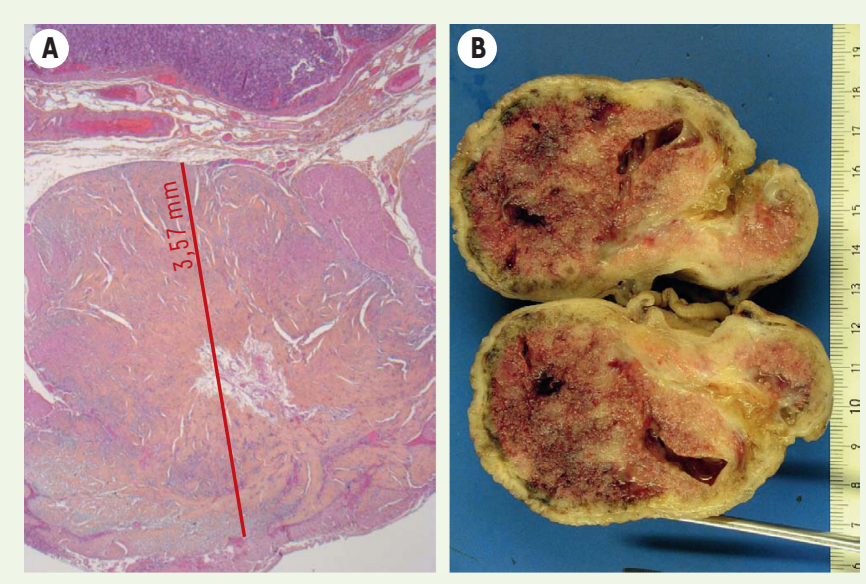

Figure 2. Exemples de GIST gastriques. La taille de la tumeur est très variable et est l'un des critères pronostiques après exérèse chirurgicale. Le risque de récidive d'une GIST gastrique de quelques millimètre ( $\boldsymbol{A}$, coloration à l'HES [hématoxyline-éosine-safran] d'une coupe histologique) sans mitose est nul, alors que celui d'une GIST de $9 \mathrm{~cm}$ ( $\boldsymbol{B}$, photographie macroscopique) avec plus de 5 mitoses $/ \mathrm{mm}^{2}$ est élevé, bien que la tumeur soit très bien limitée en périphérie.

Les micro-GIST sont des tumeurs de taille inférieure à $1 \mathrm{~cm}$. Elles sont le plus souvent de découverte fortuite (chirurgie intraabdominale pour une autre cause ou autopsie). Elles sont très fréquentes pouvant atteindre selon certaines études plus d'un quart de la population des patients de plus de 50 ans [13].

Les GIST sont de pronostic très variable, certaines ne récidivent pas après résection chirurgicale, d'autres ont une évolution métastatique rapidement catastrophique. Au moment du diagnostic, $10 \%$ des GIST sont au stade métastatique [4]. Pour les tumeurs localisées, le risque de récidive est actuellement évalué en fonction de la localisation primitive, de la taille et de l'activité mitotique (Tableau I). En fonction de ces paramètres, le risque de récidive peut être quasi nul ou dépasser $70 \%$ [14]. Un autre paramètre d'importance capitale est la rupture tumorale dans la cavité abdominale, spontanée ou peropératoire [15]. Les métastases des GIST sont initialement localisées soit au péritoine, soit au foie. Les métastases ganglionnaires sont exceptionnelles, le curage ganglionnaire n'est donc pas indiqué lors de la résection de la tumeur primitive.

\section{Mutations des gènes KIT ou PDGFRA « gain de fonction 》 dans les GIST : nature et valeur pronostique}

Les récepteurs à activité tyrosine kinase (RTK) sont des protéines transmembranaires comportant un domaine extracellulaire permettant la liaison avec un ligand spécifique, un domaine transmembranaire et un domaine intracellulaire [16]. Ce dernier présente un ou deux sites d'activité tyrosine kinase responsable(s) de la transmission du signal intracellulaire. La liaison du ligand spécifique (stem cell factor pour KIT) induit la dimérisation du récepteur, sa phosphorylation et l'activation des voies de signalisation intracellulaire. Les trois princi-

\begin{tabular}{|c|c|c|c|}
\hline \multirow{2}{*}{$\begin{array}{c}\text { Index } \\
\text { mitotique }\end{array}$} & \multirow{2}{*}{$\begin{array}{c}\text { Taille } \\
\text { tumorale }\end{array}$} & \multicolumn{2}{|c|}{ Risque de récidive } \\
\hline & & Estomac & $\begin{array}{c}\text { Intestin } \\
\text { grêle }\end{array}$ \\
\hline \multirow{4}{*}{$\leq 5$ mitoses* } & $\leq 2 \mathrm{~cm}$ & Bénin & Bénin \\
\hline & $>2$ et $\leq 5 \mathrm{~cm}$ & Très faible & Faible \\
\hline & $>5$ et $\leq 10 \mathrm{~cm}$ & Faible & Modéré \\
\hline & $>10 \mathrm{~cm}$ & Modéré & Élevé \\
\hline \multirow{4}{*}{$>5$ mitoses* } & $\leq 2 \mathrm{~cm}$ & 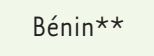 & 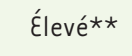 \\
\hline & $>2$ et $\leq 5 \mathrm{~cm}$ & Modéré & Élevé \\
\hline & $>5$ et $\leq 10 \mathrm{~cm}$ & Élevé & Élevé \\
\hline & $>10 \mathrm{~cm}$ & Élevé & Élevé \\
\hline
\end{tabular}

Tableau 1. Évaluation du risque de récidive des GIST après résection chirurgicale. * Le compte mitotique sur 50 champs à fort grossissement est évalué sur une surface de $5 \mathrm{~mm}^{2}$, soit 20 à 25 champs au grossissement $\times 400$ sur des microscopes récents. ${ }^{*}$ Très faible nombre de cas.

pales voies d'activation cellulaire sont RAS-RAF-MAPK (mitogen-activated protein kinase), PI3K (phosphoinositide 3-kinase)-AKT (protéine kinase B) et STAT (signal transducers and activators of transcription). Les RTK constituent la principale famille de proto-oncogènes chez l'homme. Les mécanismes responsables de la transformation peuvent être une mutation modifiant la séquence primaire du récepteur, comme c'est le cas dans les GIST, une translocation ou une amplification du locus du gène.

Les mutations des gènes KIT ou PDGFRA sont dites « gain de fonction », car elles induisent l'expression de récepteurs spontanément activés, y compris en l'absence de leurs ligands spécifiques [2, 17]. Ces mutations de KIT ou PDGFRA sont présentes dans $85 \%$ des GIST [4]. Elles respectent toujours le cadre de lecture, mais peuvent correspondre à des substitutions, des délétions, des insertions ou une combinaison de ces altérations $[4$, 18, 19]. Les mutations sont habituellement classées en fonction des exons dans lesquels elles sont localisées. Les mutations de l'exon 11 de KIT sont de loin les plus fréquentes (environ $60 \%$ ). Elles sont de nature extrêmement variable, avec plus de 150 types différents. Nous avons identifié des délétions de 3 à 63 paires de bases (débutant parfois dans l'intron), des insertions de 3 à 51 paires de bases (Figure 4) et des substitutions. Les mutations de l'exon 18 de PDGFRA sont les deuxièmes en fréquence ; elles peuvent correspondre à une mutation ponctuelle (p.D842V) ou à des délétions. Les mutations de l'exon 9 de KIT sont monomorphes et correspondent, 


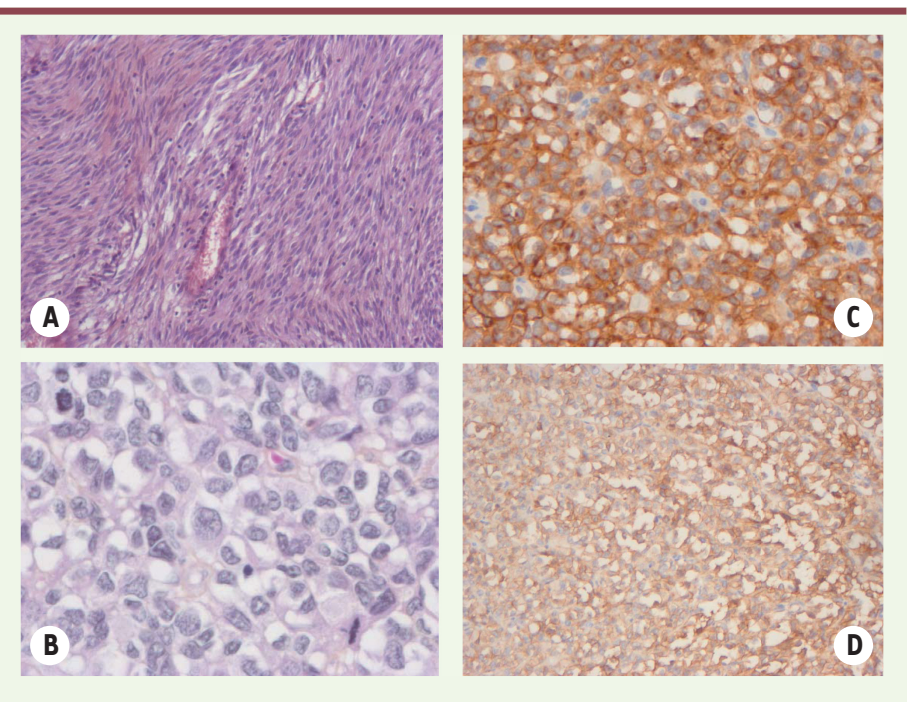

Figure 3. Histologie et immunophénotype des GIST. L'histologie des GIST peut être variable, avec notamment des aspects fusiformes $(\boldsymbol{A})$ ou épithélioïdes (B). Une immunohistochimie est nécessaire pour affirmer le diagnostic de GIST, avec une positivité pour KIT (C) et pour DOG-1 (D) dans plus de $90 \%$ des cas.

dans leur quasi totalité, à une duplication de six paires de base. Les mutations dans les exons 13 ou 17 de KIT, ou dans les exons 12 ou 14 de PDGFRA sont rares (inférieures à $1,5 \%$ des cas).

Les mutations de KIT surviennent à la phase initiale de l'oncogenèse des GIST. En effet, elles sont détectables dans les micro-GIST avec une fréquence comparable à celle des GIST de grande taille [13]. De plus, les patients exceptionnels qui présentent une mutation germinale de KIT ont une hyperplasie majeure des cellules interstitielles de Cajal qui diffuse sur toute la longueur de l'intestin grêle [11].

La détection des mutations de KIT et de PDGFRA est réalisée sur I'ADN extrait des tumeurs. En pratique courante, cet ADN est extrait à partir du bloc de tumeurs fixées et incluses en paraffine. Un contrôle morphologique est indispensable afin de s'assurer de la richesse de l'échantillon en cellules tumorales. L'ADN tumoral est ensuite amplifié par réaction de polymérisation en chaîne (PCR). Les techniques d'analyse utilisées ensuite varient suivant les laboratoires et le type de mutation recherchée.

De nombreux auteurs se sont intéressés à la valeur pronostique des mutations de KIT et PDGFRA dans les GIST [18, 20-23], et les résultats sont parfois contradictoires. Ceci s'explique par le caractère rétrospectif de la quasi totalité des études, la petite taille des effectifs (tumeurs rares), l'hétérogénéité des méthodes de détection des mutations et le biais de sélection des patients. Les principales causes de biais pouvant expliquer ces divergences sont les méthodes de détection des mutations et la date du diagnostic dans ces études rétrospectives. En effet, avant l'an 2000, beaucoup de GIST n'étaient pas diagnostiquées et, à partir de 2005, la grande majorité des patients métastatiques étaient traités avec de l'imatinib. Dans une étude prospective réalisée en France entre mai 2006 et mai 2008, incluant tous les patients diagnostiqués avec des GIST, nous avons observé des différences significatives de fréquence de certaines mutations entre les GIST localisées et les GIST métastatiques [4]. Ainsi, la mutation p.D842V de PDGFRA est détectée dans $8,5 \%$ des GIST localisées et seulement $0,8 \%$ des GIST métastatiques ( $p<0,0001$ ), ce qui suggère que cette mutation est associée à un bon pronostic. À l'opposé, la duplication de l'exon 9 de KIT est beaucoup plus fréquente dans les GIST métastatiques que localisées $(15,4 \%$ contre $4,8 \%, p<0,0001$ ) et serait de mauvais pronostic.

Les mutations de KIT apparaissent précocement au cours de l'oncogenèse, et il existe à l'évidence d'autres altérations génétiques dans les GIST, dont certaines peuvent contribuer à la croissance et/ou la diffusion métastatique des tumeurs. L'étude des GIST survenant chez les patients ayant une mutation germinale de KIT pourrait permettre d'identifier certaines des altérations potentialisant cet oncogène. Par ailleurs, les GIST présentent fréquemment des altérations chromosomiques. Ces altérations sont particulières, car elles concernent généralement des pertes ou gains de bras entiers de chromosomes. Ces altérations peuvent être détectées par des techniques à haut débit d'hybridation génomique comparative (CGH array), et semblent avoir une valeur pronostique [24, 25]. L'identification des gènes à l'origine de cette instabilité chromosomique particulière pourrait également contribuer à une meilleure connaissance de ces tumeurs et au développement de thérapies adaptées.

\section{Biochimie des formes mutées et sauvages de KIT}

La présence de mutations activatrices (gain de fonction) de proto-oncogènes est un phénomène fréquent dans les tumeurs humaines. Ainsi des mutations de KRAS (Kirsten rat sarcoma viral oncogene homolog) sont présentes dans environ $40 \%$ des carcinomes colorectaux et des mutations de BRAF (V-raf murine sarcoma viral oncogene homolog $B$ ) dans environ $50 \%$ des mélanomes. Dans la majorité des tumeurs, ces mutations oncogéniques sont présentes à l'état hétérozygote, c'est-à-dire qu'il persiste au sein des cellules tumorales un allèle non muté (sauvage) du proto-oncogène. L'allèle muté exerce alors une action dominante sur l'allèle sauvage.

Il est possible de produire des modèles in vitro de tumeurs en faisant exprimer, au sein de lignées cellulaires, des allèles mutés ou sauvages d'un oncogène. De telles études ont notamment permis de démontrer le caractère oncogénique des mutations de KIT et de PDGFRA [2, 17]. Ces modèles cellulaires ont permis également de tester in vitro la sensibilité des différentes mutations à des inhibiteurs spécifiques [26]. 


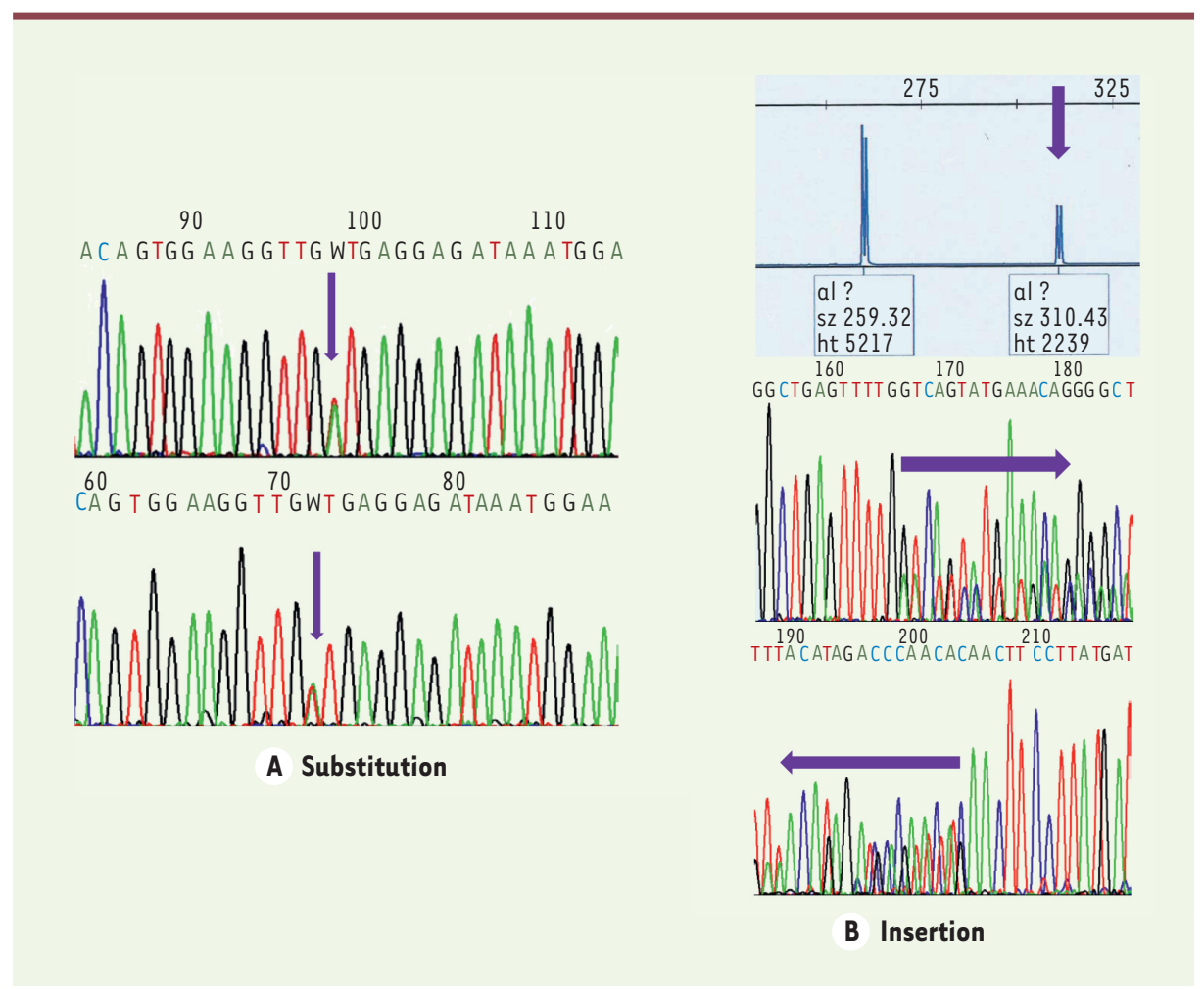

Figure 4. Mutation de KIT dans les GIST. Plus de 150 mutations différentes de KIT ou de PDGFRA ont été rapportées. Une substitution de l'exon 11 de KIT $(\boldsymbol{A})$ est identifiée par un séquençage des amplicons (flèches); les chromatogrammes des séquences désoxynucléotidiques sens (haut) et antisens (bas) sont assez similaires. Une insertion respectant le cadre de lecture dans l'exon 11 de KIT (B) est détectée par une analyse de la taille des amplicons fluorescents (haut) : le pic correspondant à l'allèle sauvage est détecté à 259 paires de bases (bp), et un pic muté correspondant à une insertion de 51 bp (flèche) est facilement détectable. L'identification précise nécessite toutefois de réaliser des séquences sens (milieu) et antisens (bas) où l'on observe une superposition des séquences des allèles sauvages et mutés (flèches horizontales).

Dans les GIST, les mutations de KIT sont également en grande majorité hétérozygotes, et nous avons montré que les deux allèles (muté et sauvage) sont coexprimés dans la quasi totalité des cas [27]. Toutefois, les mutations de KIT peuvent être à l'état homozygote dans 5 à $15 \%$ des cas, et elles sont alors associées à un mauvais pronostic $[28,29]$.

Les modèles cellulaire in vitro offrent la possibilité d'étudier si les formes mutées et sauvages de KIT ont un devenir biochimique identique (modifications post-traductionnelles, localisation et trafic intracellulaires, activation). La forme sauvage immature de KIT subit une glycosylation qui commence dans le réticulum endoplasmique rugueux et se poursuit dans l'appareil de Golgi. La protéine mature migre ensuite jusqu'à la membrane cellulaire (Figure 5), où elle est exprimée à l'état monomérique. En présence de son ligand (stem cell factor) la protéine se dimérise, se phosphoryle et active les différentes voies de signalisation intracellulaire. Ainsi, nous avons observé que des formes mutées de KIT ont un devenir biochimique très différent. En effet, elles existent majoritairement à l'état immature (non ou peu glycosylées), sont principalement localisées dans le réticulum endoplasmique ou l'appareil de Golgi, mais sont peu exprimées à la surface cellulaire [30]. De plus, les formes immatures de KIT peuvent être phosphorylées et transmettre un signal d'activation cellulaire (Figure 5). Des résultats similaires ont été obtenus par d'autres équipes [31].

Les formes mutées et sauvages de KIT ayant des devenirs biologiques très différents, et les GIST coexprimant les deux formes, il nous a semblé important d'étudier la biologie des formes mutées de KIT en comparant leur expression isolée et leur coexpression avec les formes sauvages. Dans un modèle cellulaire de fibroblastes pouvant exprimer les formes mutées ou sauvages isolément (homozygote) ou simultanément (hétérozygote), nous avons constaté que l'expression hétérozygote de KIT mutée conduisait à un comportement de la protéine beaucoup plus proche de celui de KIT sauvage que de celui des formes mutées exprimées de façon homozygote [32]. Ces résultats concernent la glycosylation, la localisation intracellulaire et la signalisation de KIT. Des résultats similaires ont été obtenus en analysant la transcription des ARN messagers et microARN dans le même modèle cellulaire [33]. II est important de noter que les analyses de sensibilité in vitro aux inhibiteurs sont pratiquement toujours réalisées sur des cellules n'exprimant que les formes mutées isolées, alors que dans la majorité des tumeurs humaines où elles sont impliquées, les protéines de type tyrosine kinase mutées sont exprimées à l'état hétérozygote.

\section{Thérapies ciblées des GIST}

Le premier succès thérapeutique avec un inhibiteur de KIT n'a été obtenu que trois ans après la découverte du rôle de cet oncogène [3]. Le succès de la thérapie ciblée avec l'imatinib dans les GIST est la première démonstration de l'efficacité d'une thérapie ciblée dans les tumeurs solides humaines et a ouvert la voie à une révolution en cancérologie. L'autorisation de mise sur le marché a été accordée dans cette indication en Europe et aux États-Unis sans nécessité d'un essai 


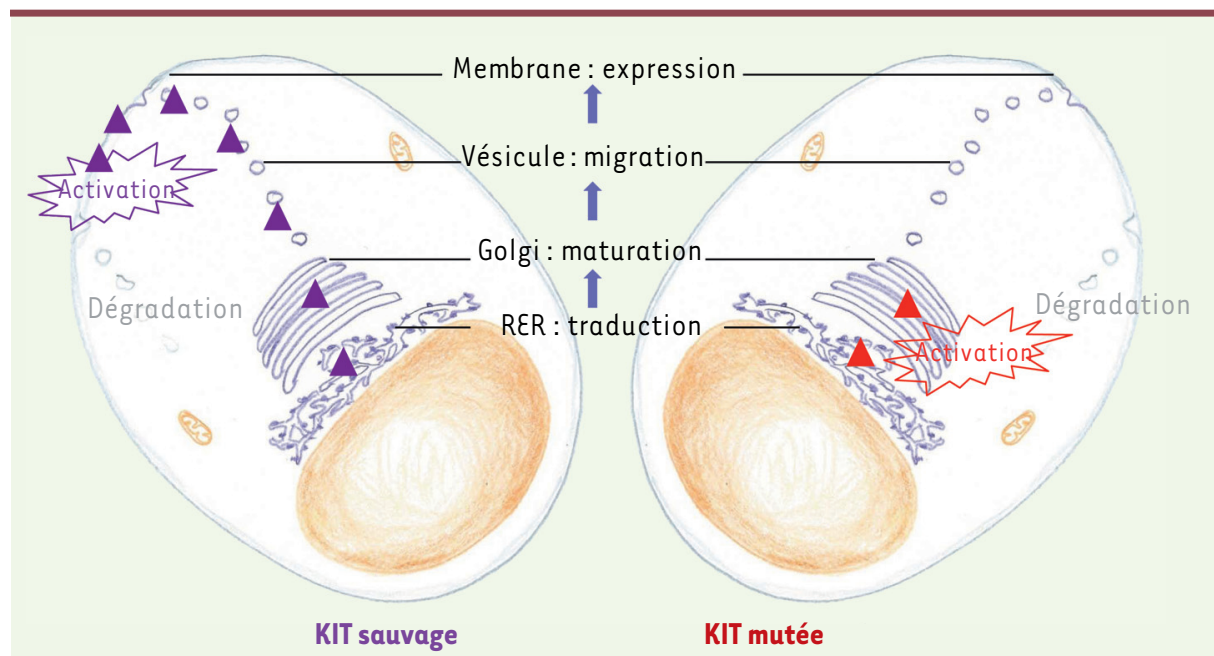

Figure 5. Biologie cellulaire des formes mutées ou sauvages de KIT. La traduction des ARN messagers en protéines et l'initiation de la glycosylation ont lieu dans le réticulum endoplasmique rugueux (RER) des cellules. Pour les formes sauvages de KIT (gauche), les protéines immatures passent ensuite dans l'appareil de Golgi où la glycosylation se poursuit. La protéine migre ensuite vers la surface cellulaire où elle est exprimées sous forme monomérique. En présence de son ligand (stem cell factor), le récepteur va se dimériser, s'activer et transmettre les signaux intracellulaires. Pour les formes mutées de KIT (droite), les étapes de glycosylation et de migration vers la surface sont perturbées car la protéine immature (peu glycosylée) est spontanément activée en position intracellulaire et en l'absence de stem cell factor. Après activation, les formes sauvages et mutées sont dégradées.

clinique randomisé. Le gain de survie obtenu par traitement avec imatinib des patients atteints de GIST métastatique est évalué à au moins trois ans. Dans cette situation, les taux de réponse tumorale sont de $45 \%$, et 72 à $76 \%$ des patients sont en vie à deux ans [34]. Le traitement adjuvant, c'est-à-dire après résection chirurgicale de la tumeur localisée, est également efficace pour diminuer le risque de récidive [35]. Malheureusement, l'imatinib ne semble pas guérir les patients. En effet, la majorité des patients ayant initialement répondu à ce traitement auront, au fil des années, un échappement thérapeutique avec apparition d'une résistance secondaire ${ }^{6}$. De plus, le groupe Sarcomes français a démontré que l'interruption du traitement était souvent associée à une reprise évolutive de la maladie y compris chez les patients en rémission complète prolongée [36]. Les résistances secondaires aux inhibiteurs des GIST mutées pour l'exon 11 de KIT sont, dans la majorité des cas, associées à l'apparition de nouvelles mutations démontrées comme responsables d'une résistance in vitro [37]. Plusieurs autres inhibiteurs de récepteurs à activité tyrosine kinase ont démontré leur efficacité thérapeutique dans les GIST. Le sunitinib a ainsi obtenu une autorisation de mise sur le marché pour le traitement de seconde ligne, après échec ou intolérance à l'imatinib, et le regorafenib a obtenu une autorisation aux États-Unis en cas d'échec des deux médicaments précédents. Le masitinib, le nilotinib et le dasatinib ont également permis d'obtenir une stabilisation ou une régression tumorale dans des essais cliniques $[38,44]$.

\footnotetext{
${ }^{6}$ Une telle résistance est également rencontrée chez les patients atteints de LMC et traités par Glivec.
}

La sensibilité à l'imatinib est variable en fonction des mutations de KIT et PDGFRA. Ceci a été observé in vitro, avec l'expression isolée des formes mutées, mais également in vivo. Ainsi, les patients métastatiques traités par une dose initiale quotidienne de $400 \mathrm{mg}$ ont une survie plus faible que ceux traités avec $800 \mathrm{mg}$ [39]. Les traitements par double dose sont donc recommandés dans cette situation. Les mutations de type p.D842V du PDGFRA sont, quant à elles, responsables d'une résistance à l'imatinib in vitro et in vivo [40]. Enfin, les mutations de l'exon 11 de KIT sont au contraire associées à une bonne réponse thérapeutique [26].

\section{Conclusions et perspectives}

Bien qu'étant les sarcomes les plus fréquents chez l'homme, les GIST n'ont été identifiées clairement que depuis une quinzaine d'année. Ces tumeurs sont ensuite devenues en quelques années le paradigme des thérapies ciblées des tumeurs solides, et ont souvent été à l'avant-garde de cette révolution en oncologie. Il reste toutefois encore de vastes champs à explorer pour mieux comprendre les mécanismes oncogéniques des protéines de type tyrosine kinase et les modes d'action des thérapies ciblées [41]. $\diamond$

\section{SUMMARY}

Gastrointestinal stromal tumors (GIST): at the forefront of targeted therapies

Although gastrointestinal stromal tumors (GIST) are the most frequent sarcomas, they were usually not diagnosed before 1998. GIST derive from interstitial cells of Cajal, and may develop along the digestive tract, mainly from stomach and small intestine. GIST are characterized by the expression of KIT (CDI17), and mutations KIT or PDGFRA are present in $85 \%$ of cases. More than 150 different types of mutations have been reported. They are responsible for a constitutive activation of these tyrosine kinase receptors, in absence of their specific ligand. Detection of these mutations may help to confirm the diagnosis or to evaluate the prognosis. The mutations also have a predictive value. Indeed patients with metastatic GIST and duplication within exon 9 of KIT 
deserve to receive twice the dose of imatinib, while GIST with PDGFRA p.D842V mutation are resistant to this drug. This review presents the main characteristics of GIST, and focus on the important insights of studies on GIST and their cell models in the field of oncology. $\diamond$

\section{LIENS D'INTÉRÊT}

L'auteur déclare n'avoir aucun lien d'intérêt concernant les données publiées dans cet article.

\section{REMERCIEMENTS}

L'auteur remercie les chercheurs, techniciens et étudiants qui ont participé aux travaux de recherche sur les GIST au sein de son équipe de recherche, en particulier J.B. Bachet, S. Brahimi, A. Gauthier, Z. Hélias, N. Lourenço, S. Tabone, N. Théou, les collègues du Groupe sarcome français et de la Fédération francophone de cancérologie digestive, ainsi que les très nombreux médecins et chirurgiens ayant contribué à l'étude MolecGIST.

\section{RÉFÉRENCES}

1. Mazur MT, Clark HB. Gastric stromal tumors, reappraisal of histogenesis. Am J Surg Pathol 1983; $7: 507-19$.

2. Hirota S, Isozaki K, Moriyama Y, et al. Gain-of-function mutations of c-kit in human gastrointestinal stromal tumors. Science $1998 ; 279: 577-80$.

3. Joensuu H, Roberts PJ, Sarlomo-Rikala M, et al. Effect of the tyrosine kinase inhibitor STI571 in a patient with a metastatic gastrointestinal stromal tumor. N EnglJ Med 2001 ; 344 : 1052-6.

4. Emile JF, Brahimi S, Coindre JM, et al. Frequencies of KIT and PDGFRA mutations in the MolecGIST prospective population-based study differ from those of advanced GISTs. Med Oncol $2012 ; 29$ : 1765-72.

5. Cassier PA, Ducimetière F, Lurkin A, et al. A prospective epidemiological study of new incident GISTs during two consecutive years in Rhône-Alpes region: incidence and molecular distribution of GIST in a European region. Br J Cancer $2010 ; 103: 165-70$.

6. Coindre JM, Emile JF, Monges G, et al. Tumeurs stromales gastro-intestinales: définition, caractéristiques histologiques, immunohistochimiques et génétiques, stratégie diagnostique. Ann Pathol $2005 ; 25: 358-85$

7. Emile JF, Scoazec JY, Coindre JM. Tumeurs stromales gastrointestinales (GIST) : quoi de neuf en 2009 ? Ann Pathol $2009 ; 29: 20-3$.

8. Maas CP, ter Haar G, van der Gaag I, Kirpensteijn J. Reclassification of small intestinal and cecal smooth muscle tumors in 72 dogs: clinical, histologic, and immunohistochemical evaluation. Vet Surg $2007 ; 36: 302-13$.

9. Miettinen M, Fetsch JF, Sobin LH, Lasota J. Gastrointestinal stromal tumors in patients with neurofibromatosis 1: a clinicopathologic and molecular genetic study of 45 cases. Am J Surg Pathol $2006 ; 30: 90-6$.

10. Pasini B, McWhinney SR, Bei T, et al. Clinical and molecular genetics of patients with the CarneyStratakis syndrome and germline mutations of the genes coding for the succinate dehydrogenase subunits SDHB, SDHC, and SDHD. Eur J Hum Genet $2008 ; 16: 79-88$.

11. Bachet JB, Emile JF. Diagnostic criteria, specific mutations and genetic predisposition in gastrointestinal stromal tumors (GISTs). Appl Clin Genet 2010 ; 3 : 1-17.

12. Miettinen M, Wang ZF, Sarlomo-Rikala M, et al. Succinate dehydrogenase-deficient GISTs: a clinicopathologic, immunohistochemical, and molecular genetic study of 66 gastric GISTs with predilection to young age. Am J Surg Pathol $2011 ; 35: 1712-21$.

13. Agaimy A, Wünsch PH, Hofstaedter F, et al. Minute gastric sclerosing stromal tumors (GIST tumorlets) are common in adults and frequently show c-KIT mutations. Am J Surg Pathol $2007 ; 31: 113-20$.

14. Miettinen $M$, Lasota J. Gastrointestinal stromal tumors: pathology and prognosis at different sites. Semin Diagn Pathol $2006 ; 23: 0-83$.

15. Joensuu H, Vehtari A, Riihimäki J, et al. Risk of recurrence of gastrointestinal stromal tumour after surgery: an analysis of pooled population-based cohorts. Lancet Oncol $2012 ; 13: 265-74$.

16. Blume-Jensen P, Hunter T. Oncogenic kinase signalling. Nature $2001 ; 411: 355-65$.

17. Heinrich $M C$, Corless $C L$, Duensing $A$, et al. PDGFRA activating mutations in gastrointestinal stromal tumors. Science $2003 ; 299: 708-10$.

18. Emile JF, Théou N, Tabone $S$, et al. Clinicopathologic, phenotypic, and genotypic characteristics of gastrointestinal mesenchymal tumors. Clin Gastroenterol Hepatol $2004 ; 2$ : 597-605.

19. Rubin BP, Heinrich MC, Corless CL. Gastrointestinal stromal tumour. Lancet 2007 ; 369 : 1731-41.

20. Taniguchi M, Nishida T, Hirota $S$, et al. Effect of $\mathrm{c}-k$ it mutation on prognosis of gastrointestinal stromal tumors. Cancer Res $1999 ; 59: 4297-300$.

21. Wardelmann $\varepsilon$, Losen I, Hans V, et al. Deletion of Tryp-557 and Lys-558 in the juxtamembrane domaine of the $c$-kit protooncogene is associated with metastatic behavior of gastrointestinal stromal tumors. Int J Cancer $2003 ; 106: 887-95$.
22. Martin J, Poveda A, Llombart-Bosch A, et al. Deletions affecting codons 557558 of the c-KIT gene indicate a poor prognosis in patients with completely resected gastrointestinal stromal tumors: a study by the Spanish Group for sarcoma research (GEIS). J Clin Oncol $2005 ; 23: 6190-8$.

23. Bachet JB, Hostein I, Le Cesne A, et al. Prognosis and predictive value of KIT exon 11 deletion in GISTs. BrJ Cancer 2009; 101 : 7-11.

24. Chibon F. Prédiction du risque métastatique dans les sarcomes : une nouvelle approche. Med Sci (Paris) $2011 ; 27: 22-4$.

25. Lagarde P, Pérot G, Kauffmann A, et al. Mitotic checkpoints and chromosome instability are strong predictors of clinical outcome in gastrointestinal stromal tumors. Clin Cancer Res $2012 ; 18: 826-38$.

26. Heinrich MC, Corless CL, Demetri GD, et al. Kinase mutations and imatinib response in patients with metastatic gastrointestinal stromal tumor. J Clin Oncol $2003 ; 21: 4342-9$.

27. Théou N, Tabone S, Saffroy R, et al. High expression of both mutant and wild-type alleles of $\mathrm{c}$-kit in gastrointestinal stromal tumors. Biochim Biophys Acta 2004 ; 1688 : 250-6.

28. Lasota J, vel Dobosz AJ, Wasag B, et al. Presence of homozygous KIT exon 11 mutations is strongly associated with malignant clinical behavior in gastrointestinal stromal tumors. Lab Invest 2007 ; 87 : 1029-41.

29. Emile JF, Bachet JB, Tabone- glinger S, et al. GIST with homozygous KIT exon 11 mutations. Lab Invest $2008 ; 88: 456-7$.

30. Tabone- $\varepsilon$ glinger $\mathrm{S}$, Subra F, દl Sayadi H, et al. KIT mutations induce intracellular retention and activation of an immature form of the KIT protein in gastrointestinal stromal tumors. Clin Cancer Res 2008 ; 14 : 2285-94.

31. Bougherara H, Subra F, Crépin R, et al. The aberrant localization of oncogenic kit tyrosine kinase receptor mutants is reversed on specific inhibitory treatment. Mol Cancer Res $2009 ; 7: 1525-33$.

32. Brahimi-Adouane S, Bachet JB, Tabone-Eglinger S, et al. Effects of endoplasmic reticulum stressors on maturation and signaling of hemizygous and heterozygous wild-type and mutant forms of KIT. Mol Oncol 2012 ; doi : pii: S1574-7891(12)00106-8.

33. Bachet JB, Tabone-Eglinger S, Dessaux S, et al. Gene expression patterns of hemizygous and heterozygous KIT mutations suggest distinct oncogenic pathways: a study in NIH3T3 cell lines and GIST samples. PlosOne 2013 ; 8 : e61103.

34. Blanke CD, Rankin C, Demetri GD, et al. Phase III randomized, intergroup trial assessing imatinib mesylate at two dose levels in patients with unresectable or metastatic gastrointestinal stromal tumors expressing the kit receptor tyrosine kinase: S0033. J Clin Oncol 2008; $26: 626-32$.

35. DeMatteo RP, Ballman KV, Antonescu CR, et al. Adjuvant imatinib mesylate after resection of localised, primary gastrointestinal stromal tumour: a randomised, double-blind, placebo-controlled trial. Lancet 2009 ; 373 : 1097-104.

36. Le Cesne A, Ray-Coquard I, Bui BN, et al. Discontinuation of imatinib in patients with advanced gastrointestinal stromal tumours after 3 years of treatment: an open-label multicentre randomised phase 3 trial. Lancet Oncol $2010 ; 11: 942-9$.

37. Heinrich MC, Corless CL, Blanke CD, et al. Molecular correlates of imatinib resistance in gastrointestinal stromal tumors. J Clin Oncol 2006 ; 24 : 476474.

38. Kim हJ, Zalupski MM. Systemic theray for advanced gastrointestinal stromal tumors: Beyond Imatinib. J Surg Oncol 2011 ; 104 : 901-6.

39. Gastrointestinal stromal tumor meta-analysis group (MetaGIST). Comparison of two doses of imatinib for the treatment of unresectable or metastatic gastrointestinal stromal tumors: a meta-analysis of 1,640 patients. J Clin Oncol $2010 ; 28$ : 1247-53.

40. Cassier PA, Fumagalli $\varepsilon$, Rutkowski P, et al. Outcome of patients with platelet-derived growth factor receptor alpha-mutated gastrointestinal stromal tumors in the tyrosine kinase inhibitor era. Clin Cancer Res 2012 $18: 4458-64$.

41. Blay JY. Le futur des thérapeutiques ciblées en oncologie : trouver les cibles, traiter tôt et au long court. Med Sci (Paris) 2007 ; 23 : 1073-4.

42. Turhan $A$. L'imatinib mésylate : une révolution dans le traitement de la leucémie myéloïde chronique. Med Sci (Paris) 2003 ; $19: 667-8$.

43. Favier J, Gimenez-Roqueplo AP. La génétique des paragangliomes et des phéochromocytomes. Med Sci (Paris) $2012 ; 28: 625-32$.

44. Etienne $G$, Huguet $F$. Leucémie myéloïde chronique et thérapeutiques ciblées. Bientôt l'embarras du choix ? Med Sci (Paris) 2010 ; 26 : 911-4.

\section{TIRÉS À PART \\ J.-F. Émile}

